\title{
Severe herpes simplex oral mucositis in a patient with non-Hodgkin's lymphoma after autologous stem cells transplantation - Case Report
}

\author{
Magdalena Kostyra', Joanna Mańko², Jolanta Wojciechowicz'1, Marek Hus², Tomasz Tomaszewski ${ }^{1}$ \\ ${ }^{1}$ Maxillofacial Surgery Department, Medical University of Lublin \\ 2 Department of Haematooncology and Bone Marrow Transplantation, Medical University of Lublin
}

Kostyra M, Mańko J, Wojciechowicz J, Hus M, Tomaszewski T. Severe herpes simplex oral mucositis in a patient with non-Hodgkin's lymphoma after autologous stem cells transplantation - Case Report. J Pre-Clin Clin Res. 2017; 11(1): 38-41. doi: 10.26444/jpccr/75146

\section{Abstract}

The study presents the case of a 25-year-old patient with the diagnosis of diffuse large B cell lymphoma treated autologously with haematopoietic stem cells transplantation. During the period of aplasia after chemotherapy severe sore lips, mouth and esophagus herpes simplex mucositis developed and is successfully treated with foscarnet. The clinical course, localized causal and symptomatic treatment, along with the therapy imaging, are presented.

\section{Key words}

herpes simplex mucositis, non-Hodgkin's lymphoma, autologous haematopoietic stem cells transplantation, foscarnet

\section{INTRODUCTION}

Mucositis is inflammation of the mucosus membrane, known as mucosal barrier injury (MBI), which can be located within the oral cavity - oral MBI (OMBI), or further sections of the gastrointestinal tract - gut MBI (GMBI). Mucositis occurs in approximately $76-89 \%$ of patients with haematologic malignancies treated with intensive chemotherapy, including conditioning myeloablative therapy prior to autologous haematopoietic stem cell transplantation (auto-HSCT). The occurrence of MBI depends on many factors: the protocol used in chemotherapy, radiotherapy, completed and concomitant diseases, age, gender, health and oral microflora, salivary gland function, or addictions. Pathological processes occurring in the course of MBI may be benign inflammatory lesions, erosions, or difficult-to-heal ulcers, which has a significant impact on the quality of the patient's life. Furthermore, due to violation of natural mucosal barrier by breaking the continuity of tissues and impairment of host defence processes, MBI favours the occurrence of secondary infections - viral, fungal and bacterial, which can be severe and life-threatening.

Clinically, changes in the course of oral mucositis are frequently classified according to the Oral Toxicity Scale (OTS) developed by the World Health Organization (WHO). The WHO scale takes into account an assessment of anatomical changes, clinical symptoms and functional disorders in the course of mucositis, in 5 grades of severity. According to OTS, grade 0 - no lesions, 1 - erythema \pm pain, 2 - erythema, ulceration, the patient can take solid foods, 3 - ulcers, with massive oedema, the patient is unable to eat solid foods, 4 - advanced mucositis prevents the of any food.

The cytostatics having a particularly toxic effect on the mucous membrane and responsible for the occurrence of MBI

Address for correspondence: Magdalena Kostyra, Maxillofacial Surgery Department, Medical University of Lublin, Poland

E-mail: magda.kostyra@gmail.com

Received: 26 October 2016; accepted: 24 April 2017 include: folic acid antagonists (methotrexate), anthracycline (idarubicin, daunorubicin, doxorubicin), pyrimidine analogs (5-fluorouracil, cytosine arabinoside), alkylating agents (high dose melphalan, busulfan, cyclophosphamide), cisplatin and carboplatin, topoisomerase II inhibitors (etoposide, irinotecan), and purine analogues (fludarabine, cladribine). Most of these drugs reach the mucosa via blood vessels. Methotrexate, and etoposide are also secreted into the saliva and can therefore directly damage the mucosa. Damage to the protective barrier of the oral mucosa by cytotoxic agents results in its high susceptibility to the very diverse microbiotic oral environment, including gram-positive, gram-negative bacteria, fungi, in particular from the group of Candida, viruses, as well as exogenous microorganisms. Viral infections, being a complication of myeloablative therapy, are a very serious problem for both health and the economy. Their effects include a severe course of inflammation of the mucous membranes with massive ulcers, severe pain preventing the intake of food and liquids, requiring parenteral nutrition, analgesic treatment and contribute to longer hospitalization and increased costs of treatment.

Herpes simplex virus -1 (HSV-1) is present within the oral mucosa and, together with other viral pathogens (Epstein-Barr virus, EBV, cytomegalovirus, CMV), may be an important pathognomonic factor in the development of OMI. Herpes viruses are characterized by high resistance to antiviral treatment and are an important negative factor in the etiology and course of OMI.

Clinically, infection with HSV-1 can manifest itself in the form of severe mucositis, inflammation of the lungs or gastrointestinal tract [1]. Primary infection with HSV-1 usually occurs in childhood and exists in a latent form in the trigeminal ganglia. In adults, the factors causing reactivation of viral replication include: sunlight, stress, trauma, fever, immunosuppression. Each replication process manifests itself in the form of recurrent infection with herpes labialis, or sometimes erosions in the mouth. In the case of herpes labialis, usually the first symptom of changes occurring on 
the mucocutaneous border is itching, burning, tingling, or pain. Clinically, herpetic lesions are manifested in the form of a spot, then a lump on the base, wherein within 48 hours numerous vesicles are formed. After 72-96 h, changes take the form of pustules, crusts, which heal without scarring. In the mouth, erosions may occur locating the hard palate or gums that disappear after 1-2 weeks. In the case of immuno-suppressed patients, changes initially take the form of proliferating erosion-ulcerative changes, and besides the typical location, they are often also on the dorsal side of the tongue [2].

\section{CASE REPORT}

A 25-year old male patient diagnosed with diffuse large B-cell lymphoma (DLBCL), an originally resistant form of the disease, after 3 lines of chemotherapy and radiotherapy of neck, was qualified to high dose chemotherapy procedure supported with autologous hematopoietic stem cell transplantation. In the conditioning, R-BEAM protocol was used (rituximab, BiCNU, etoposide, cytosine arabinoside, melphalan) in maximum doses because of the high activity of the disease and resistance to current treatment. Acyclovir was used in the prophylaxis of viral infections.

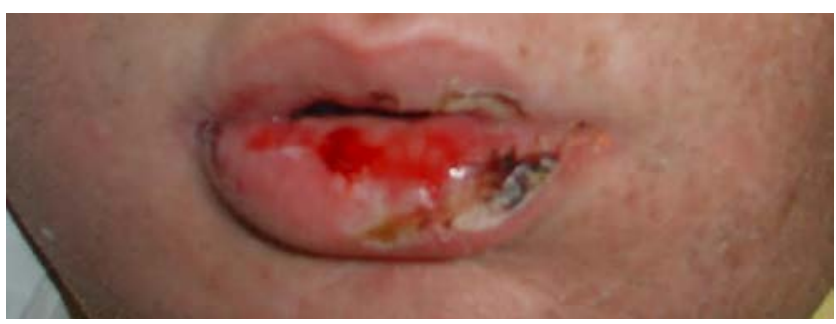

Figure 1. The clinical manifestation of infection with HSV-1 after autoHSCT (+12). First symptoms

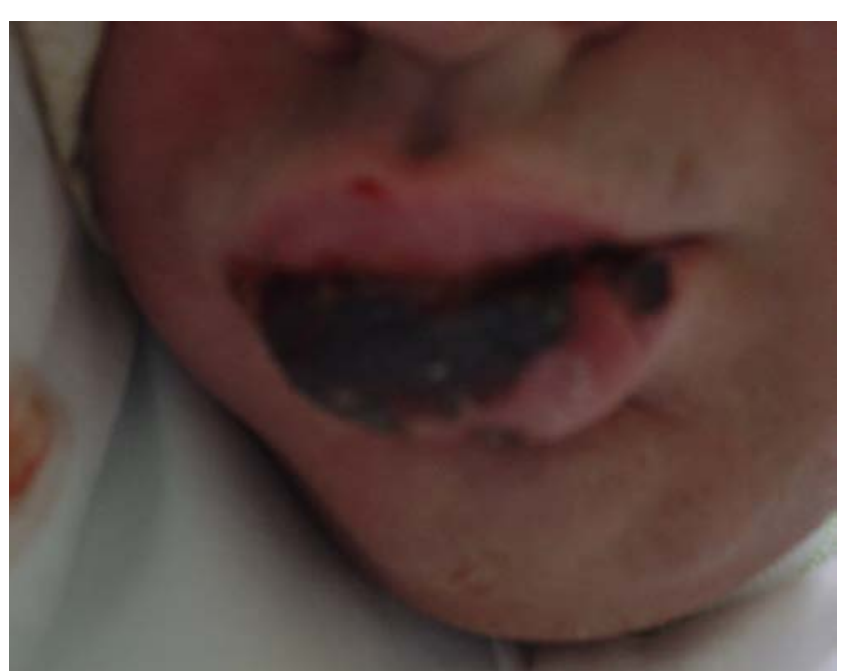

Figure 2. The clinical manifestation of infection with HSV-1 after autoHSCT (+34). Advanced symptoms

In the dental examination before the conditioning procedure, there were no pathological lesionss on the mucosa of the lips, cheeks, hard and soft palate, floor of the mouth; only the tongue ridge was covered with a thin, clear coating ( $2^{\text {nd }}$ degree Winkle tongue coating), and the gum showed a slight state of inflammation manifested by

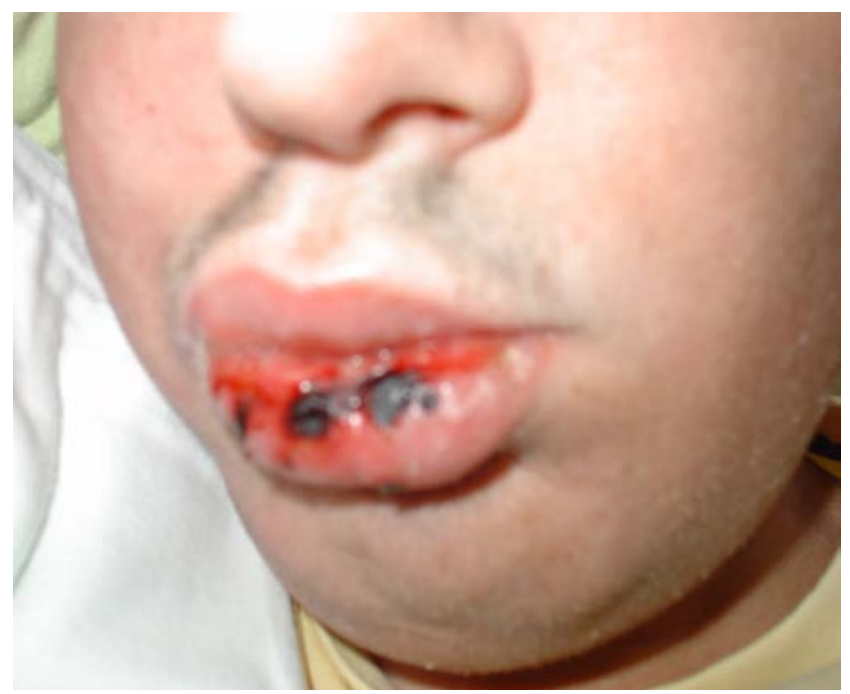

Figure 3. The clinical manifestation of infection with HSV-1 after autoHSCT $(+40)$. Period of healing

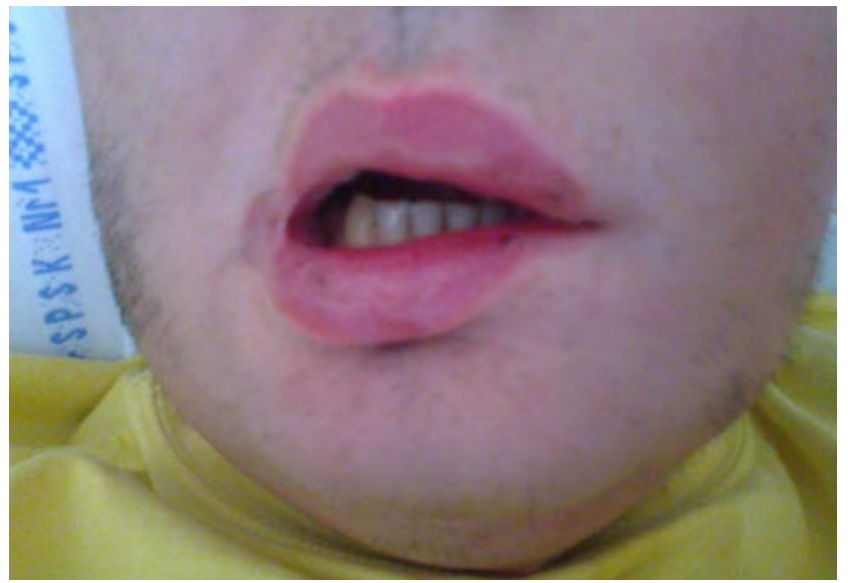

Figure 4. Clinical view after autoHSCT complicated by HSV-1. Three months later

bleeding on probing (gingival sulcus bleeding index [GSBI], $1^{\text {st }}$ degree). Clinical examination and evaluation of the orthopantomographic images did not reveal the presence of dead teeth, nor endodontically treated teeth. For the assessment of oral hygiene, Oral Hygiene Index (OHI) by Greene and Vermillion was used, whose average for the dental calculus was 0 and for plaque - 1 . In the extraoral examination, there were no pathological changes on upper and lower vermilion borders.

During prolonged marrow aplasia after chemotherapy, inflammation of the oral mucosa, lips, throat and oesophagus of a mucositis type was observed. Grade 4 of mucositis was diagnosed according to the $\mathrm{WHO}$ grading scale, requiring administration of narcotic analgesics and application of parenteral nutrition for a period of 38 days. Diagnosis of severe MBI revealed the presence of HSV-1 in blood by PCR test; viral infections CMV and EBV were excluded.

Infection with HSV-1 proceeded with simultaneous, deep bone marrow suppression and prolonging recovery of all cell lines. This resulted in the need to use a number of transfusions of red blood cell concentrates (16 units) and platelet concentrates (16 packs).

The patient required the use of broad-spectrum antibiotics therapy, antifungal agents (fluconazole, caspofungin) and 
intravenous immunoglobulin preparations. In the treatment of herpetic mucositis, due to marrow aplasia, foscarnet was applied, instead of the reference acyclovir. Improvement of the general condition of the patient, together with the healing of erosions and ulcerative chan ges, was obtained after 7 days of treatment with foscarnet. At the same time, normal bone marrow regeneration with improved blood counts were observed and resignation of the need for transfusion of blood products. The patient was discharged from hospital on day 44 after bone marrow transplantation. The healing period of lesions in the oral cavity after discharge from the hospital was another 28 days, but it allowed for liquid alimentation.

This patient with the WHO Grade 4 mucositis was unable to open his mouth, chich rendered clinical evaluation of lesions in the oral cavity considerably difficult. Inflammatory lesions in the course of infection with HSV-1 also affected almost the entire surface of the lower vermilion border. They took the form of bleeding erosions and ulcers, which had a tendency to proliferate, forming large scabs. Transformation of inflammatory lesions in the patient proceeded without follicular and vesicular phases. During hospitalization, advanced mucosal inflammation and pain in the oral cavity (assessed by the patient at the level of 6-8 in a 10-point visualanalog scale of subjective assessment of pain) limited the implementation of full hygenisation of the oral cavity. The patient was not able to brush his teeth. Only on day 14 after auto-transplantation, a lotion containing a mixture of drugs: benzocaine, neomycin, hydrocortisone and nystatin, could be introduced, as well as the use of Corsodyl and Fungizone, followed by Caphosol.

\section{DISCUSSION}

Viral infections of the oral mucosa are a serious complication of chemotherapy and radiotherapy in patients with haematologic malignancies $[3,4]$. Herpes simplex viruses are detected in $58-70 \%$ of haematology patients treated with intensive chemotherapy [5]. The response of HSV-1 is activated specifically in the $3^{\text {rd }}$ and $4^{\text {th }}$ grade of OMI severity, with concomitant neutropenia, leading to formation of endothelial vesicles that rupture, releasing exudate, comprising among others, fibrin forming pseudo-membranes, which makes the course of OMI extremely aggressive and resistant to treatment. Chen et al. reported that antifungal drugs could potentially increase the risk of infection with HSV-1 in the course of OMI of about 1.02-fold [5]. Figliolia et al. analyzed a group of 169 children with acute lymphocytic leukemia (ALL) in whom OMI was diagnosed in 77 patients (46\% of cases), of whom 23 patients were diagnosed with HSV-1. Mendon et al. studied a group of 71 paediatric patients diagnosed with ALL for the presence of HSV-1 and other microorganisms. Determination of HSV-1 was performed on the $14^{\text {th }}$ and $56^{\text {th }}$ day after introduction of high dose chemotherapy in the GBTLI ALL-99 protocol. The presence of virus was detected in $14.3 \%$ of cases on the $14^{\text {th }}$ day of the test, and in $6.3 \%$ of cases on day 56. The author concluded that carrying of HSV-1 is a factor exacerbating the intensity of changes in the course of oral mucositis type, but OMI also triggers the virus replication by cytokines secreted by the damaged cells. $\mathrm{HSV}$-seropositive patients more often are exposed to more severe OMI (WHO grades 3 and 4) [6]. Beek et al. evaluated the presence of HSV, EBV and CMV in 49 patients treated in 2006-2009 at the University Medical Centre in Leiden, Holland, with haematological malignancies qualified for HSCT. The carriers of of HSV- 1 were found in $80 \%$ of patients before HSCT. Researchers state that the persistence of HSV1 infection in spite of the treatment with acyclovir, affected approximately $18 \%$ of patients, and probably was the result of a mutation located in a TK gene (thymidine kinase) $[7,8]$. Research by Andrei et al. has shown that up to $50 \%$ of HSV-1 strains clinically resistant to acyclovir therapy may contain mutations of this type [9]. It seems that the most appropriate management is to carry out tests in patients for the presence of HSV-1 before initiation of chemotherapy and the use of antiviral prophylactic therapy [10]. The drug of choice in the prevention of HSV-1 in patients undergoing HSCT is acyclovir, administered orally in a daily prophylactic dose of $600-1200 \mathrm{mg}$, or intravenously $250 \mathrm{mg} / \mathrm{m}^{2}$ every 12 hours [1]. The treatment of an active HSV-1 infection consists in administration of intravenous acyclovir at a dose of $5 \mathrm{mg} / \mathrm{kg}$ body weight, every 8 hours for 7-10 days [11]. It becomes ineffective in the case of mutation of the genome of HSV1 virus consisting in deficiency of thymidine kinase [10]. Alternative drugs for resistance to acyclovir are: foscarnet, valganyclovir and cidofovir, which act independently of the level of thymidine kinase $[1,11,12]$. Foscarnet is used at a dose of $60 \mathrm{mg} / \mathrm{kg} /$ body weight every 12 hours i.v. or $40 \mathrm{mg} / \mathrm{kg} /$ body weight every 8 hours for 7-21 days until complete healing of lesions. Cidofovir is recommended at a dose of $5 \mathrm{mg} / \mathrm{kg} /$ body weight, once a week for 2 weeks of treatment [11]. Acyclovir prophylaxis is indicated in seropositive patients. For these patients, the lack of antiviral prophylaxis causes infection in over $80 \%$ of patients. Routine use of acyclovir in HSV seronegative patients is not advisable, since a prophylactic dose of this drug contributes to the development of strains resistant to treatment [1]. Research by Frobert et al. showed that of 62 patients undergoing alloHSCT due to haematological malignancies in whom pharyngeal swab confirmed the presence of of HSV-1, the resistance to acyclovir was seen in $42 \%$ of patients [13].

\section{CONCLUSION}

It is extremely important to prepare the patient for bone marrow graft, not only from the general-medical, but also dental point of view, involving the sanation and hygenisation of the mouth. Caring for these elements, as well as compliance with the regime of transplantation procedure, the use of antiviral, antibacterial, antifungal prophylaxis, however, does not guarantee the absence of complications of a mucositis type. In the case of severe course of the oral mucositis type, one should keep in mind that the reason, among others, May be coexisting HSV-1 infection. In some cases, patients eligible for bone marrow transplantation should have marked concentration of HSV antibodies, the IgG type, before starting the procedure despite the fact that such a procedure is not standard. Furthermore, the presence of new strains of HSV-1 resistant to acyclovir treatment remains a major therapeutic problem.

In the described case, herpes mucositis in a patient led to extensive ulcerative changes requiring long-term parenteral nutrition, use of narcotic analgesics, anti-infectives and significantly prolonged bone marrow recovery time, hospital stay, and thus increased the cost of treatment. 


\section{REFERENCES}

1. Kedia S, Acharya PS, Mohammad F, Nguyen H, Asti D, Mehta S, Pant M, Mobarakai N:Infectious Complications of Hematopoietic StemCell Transplantation. J Stem Cell Res Ther (2013)S3:002

2.Scully C.:Choroby jamy ustnej. Diagnostyka i leczenie. Chapter 29. Infekcje wirusami Herpes.(2004) 342-358.

3. Djuric C, Jankovic L, Jovanovic T, Pavlica D, Brkic S, Knezevic A Markovic D, Milasin J:Prevalence of oral herpes simplex virus reactivation in cancer patients: a comparison of different techniques of viral detection. J Oral Pathol Med (2009);38(2):167-73.

4. Haverman TM,Raber-Durlacher JE,Rademacher WMH, Vokurka S, Epstein JB, Huisman C, Hazenberg MD, de Soet JJ, de Lange J, Rozema FR:Oral complications in hematopoietic stem cell recipients: the role of inflammation. Mediators of Inflammation. Volume 2014 (2014), Article ID 378281, 18 pages

5. Chen YK, Hou HA, Chow JM, Chen YC, Hsueh PR, Tien HF: The impact of oral herpes simplex virus infection and candidiasis on chemotherapyinduced oral mucositis among patients with hematological malignancies. Eur J Clin Microbiol Infect Dis (2011); 30: 753-759.

6. De Mendona RMH, De Arajo M, Levy CE, Morari J, Silva RA, Yunes JA, Brandalise SR: Prospective evaluation of HSV, Candida spp., and oral bacteria on the severity of oral mucositis in pediatric acute lymphoblastic leukemia. Support Care Cancer (2012);20:1101-1107.

7. Van der Beek MT, Laheij AMG., Raber-Durlacher JE, Von dem Borne PA, Wolterbeek R., Van der Blij-de Brouwer CS, Van Loveren C, Class ECJ, Kroes ACM, De Soet JJ, Vossen ACTM: Viral loads antiviral resistance of herpesviruses and oral ulcerations in hematopoietic stem cell transplant recipients. Bone Marrow Transplantation (2012); 47:1222-1228.

8. Harris W, Collins P, Fenton RJ, Snowden W, Sowa M, Darby G: Phenotypic and genotypic characterization of clinical isolates of herpes simplex virus resistant to aciclovir. Journal of General Virology (2003);84:1393-1401

9. Andrei G, Balzarini J, Fiten P, De Clercq E, Opdenakker G, Snoeck R:Characterization of Herpes Simplex Virus Type 1 Thymidine Kinase Mutants Selected under a Single Round of High-Dose Brivudin. J Virol May (2005);79(9): 5863-5869

10. Atalla A, Maiolino A,Guimarães MA, Guimarães AC, Nucci M: A Non-Randomized Comparative Study Using Different Doses of Acyclovir to Prevent Herpes Simplex Reactivation in Patients Submitted to Autologous Stem Cell Transplantation. The Brazilian Journal of Infectious Diseases (2005);9(3):330-335

11. Styczynski J, Reusser P, Einsele H, Camara R, Cordonnier C, Ward KN, Ljungman P, Engelhard D: Management of HSV, VZV and EBV infections in patients with hematological malignancies and after SCT: guidelines from the Second European Conference on Infections in Leukemia. Bone Marrow Transplantation (2009);43:757-770

12. Blot N, Schneider, Young P, Janvresse C, Dehesdin D, Tron P, Vannier JP: Treatmant of an acyclovir and foscarnet- resistant herpes simplex virus infection with cidofovir in a child after an unrelated bone marrow transplant. Bone Marrow Transplantation (2000);26:903-905

13. Frobert E, Sobh M, Morfin F, Lubussiere H, Ducastelle S, Gillis L, Barraco F, Escuret V, Thomas X, Nicolini FE, Lina B, Michallet M:Oral Mucositis After Allogeneic Hematopoietic Stem Cell Transplantation: Important Impact Of The Presence Of Aciclovir-Resistant Herpes Simplex Virus (HSV-1) On Its Occurrence. Blood J (2013); 122(21):1048

14. Neofytos D, Horn D, Anaissie E, Steinbach W, Olyaei A, Fishman J, Pfaller M, Chang C, Webster K, Marr K: Epidemiology and outcome of invasive fungal infection in adult hematopoietic stem cell transplant recipients: Analysis of multicenter prospective antifungal therapy (PATH) alliance registry. Fungal Infection in HSCT Recipients. Clinical Infectious Diseases (2009);48:265-273. 